\title{
THE EVALUATION OF CERTAIN INFINITE INTEGRALS INVOLVING PRODUCTS OF BESSEL FUNCTIONS: A CORRELATION OF FORMULA
}

\author{
BY \\ MARK T. HANSON AND IGUSTI W. PUJA \\ Department of Engineering Mechanics, University of Kentucky, Lexington, $K Y$
}

\begin{abstract}
This analysis evaluates certain infinite integrals continuing products of Bessel functions of integer order, an exponential and a power. The integrals considered here have been previously evaluated in the literature in two different forms. In one instance they have been written in terms of complete elliptic integrals of the first, second, and third kind. Some of these integrals have also been evaluated in terms of a Legendre function of the second kind and a complete elliptic integral of the third kind. A recent result in elasticity obtained by the authors has led to a new form for the evaluations of these integrals. The integrals are still evaluated in terms of complete elliptic integrals; however, a new modulus (and parameter for the complete elliptic integral of the third kind) is used. The new form used for the complete elliptic integral of the third kind allows the integral evaluations to be written in a more convenient form than previously given. The new form for the complete elliptic integral of the third kind is also utilized in the evaluations using the Legendre function of the second kind. The new forms to the integral evaluations derived presently are correlated with existing results in the literature.
\end{abstract}

1. Introduction. The development of integral transforms has led to great advances in problem-solving capabilities within the field of mathematical physics. In particular, the Hankel transform has found a wide usage for solving problems where the form of the boundary conditions naturally leads to a cylindrical coordinate system (Sneddon, 1972). Well-known examples of this in potential theory include determining the potential for the circular ring or disc of unit charge. Present interest stems from the field of linear elasticity where Hankel transforms have been extensively used to solve many types of boundary-value problems (Sneddon, 1951). In some instances where Hankel transforms are used, the unknown transform function turns out to be a Bessel function of integer order. In this case the inverse transform is an infinite integral containing a product of two Bessel functions of integer order, an exponential and a power. Generally there are three parameters in these integrals, one in the argument of the exponential and one in the argument of each Bessel function.

Received October 11, 1994.

1991 Mathematics Subject Classification. Primary 33A25; Secondary 73C35, 31B10. 
For an isotropic half space subjected to a uniform pressure loading over a circular area on the surface, Terazawa (1916) showed that a Hankel transform solution leads to integrals of the type noted above. It was shown by Muki (1960) that a uniform shear loading also leads to integrals of this type. Apparently the first systematic discussion of these integrals was presented by Eason et al. (1955). Eight integrals of this type were evaluated in terms of complete elliptic integrals of the first, second, and third kind. Recurrence relations were also derived which could be used to evaluate additional integrals. They used the forms of the complete elliptic integrals which were tabulated by Heuman (1941). Hence Heuman's Lambda function was used in place of the complete elliptic integral of the third kind. They also noted a result by Sura-Bura (1950) and showed that their expression for this particular integral was equivalent.

These inverse Hankel transform integrals generally represent some physical entity. For example, in the elasticity problem of uniform pressure loading over a circular area on a half space, the integrals represent the elastic displacement and stress fields throughout the body and contain three parameters. The parameter in the exponential is the $z$-coordinate directed into the half space. There is also a different parameter in the argument of each Bessel function; one represents the polar radius in cylindrical coordinates and the other is the radius of loading. An interesting feature is noticed when examining the results in Eason et al. (1955). It is apparent that when the integral evaluation does not contain Heuman's Lambda function, only one expression is needed to evaluate the integral for all values of the parameters. However, when the integral evaluation also contains Heuman's Lambda function, two different expressions are given for the integral evaluation depending on the relative magnitude of the polar radius with respect to the radius of loading. Since the elastic field inside the half space is continuous and has continuous derivatives, it is troublesome that the expressions are different depending on being inside or outside the radius of loading. Intuition would lead one to expect a single expression which is valid at every point in the half space.

In a recent paper (Hanson and Puja, 1996) the authors reconsidered the problem of uniform normal and shear loading on the surface of a half space. This new solution was derived for a transversely isotropic material. Rather than use Hankel transforms, the method developed by Love (1929), who also considered the isotropic half space, was utilized. The solution to this problem has provided a new relation between different forms of the complete elliptic integral of the third kind. Using this result, the elastic field was written as a single expression for all values of the coordinates. The new forms for the complete elliptic integrals are used below to re-evaluate the results in Eason et al. (1955) in more convenient expressions.

The present results are broken down into several small sections which are now outlined. In Sec. 2, a particular integral of the class considered here is related to the linear elastic potential function in Hanson and Puja (1996). This will allow certain integral evaluations to be written down directly from the results in their paper. Section 3 presents the definitions of the complete elliptic integrals along with the new modulus and parameter. The forms used by Eason et al. (1955) are discussed in Sec. 4 and the relations between their forms and those used presently are considered in Sec. 5. Section 6 presents differentiation formulas necessary to obtain some of the results in the present paper. Section 
7 gives the new evaluations to certain integrals and they are compared to Eason et al. (1955) in Sec. 8. The result derived by Sura-Bura (1950) is compared in Sec. 9. Sections 10 and 11 give new evaluations to additional integrals.

As a final point it is noted that a few integrals of the class considered here have also been evaluated in terms of a Legendre function of the second kind (Erdelyi, 1954; Byrd and Friedman, 1971). Some of the integrals considered here have been evaluated in terms of this Legendre function and the complete elliptic integral of the third kind by Hasegawa et al. $(1992 \mathrm{a}, \mathrm{b})$ who investigated some axisymmetric problems in linear elasticity. In these last two papers, the choice of the modulus and parameter in the complete elliptic integral of the third kind is identical to Eason et al. (1955). Thus their expressions are subject to the same comments as above. In Sec. 12 these integrals are evaluated with the new modulus and parameter for the complete elliptic integral of the third kind, along with the Legendre function of the second kind.

2. Alternative representation. The object of this paper is to evaluate some infinite integrals involving products of Bessel functions, an exponential and a power. Using the definition of Eason et al. (1955), these integrals are denoted as

$$
I(\mu, \nu ; \lambda)=\int_{0}^{\infty} \xi^{\lambda} J_{\mu}(a \xi) J_{\nu}(\rho \xi) e^{-\xi z} d \xi
$$

where $a, \rho$, and $z$ are nonnegative quantities. In the present paper we will only consider integer values of $\mu, \nu$, and $\lambda$. In this case the integral can be evaluated in terms of complete elliptic integrals of the first, second, and third kinds.

As the starting point for the present method, an alternative expression for $I(\mu, \nu ; \lambda)$ is sought for a particular set of values of $\mu, \nu$, and $\lambda$. We start with the integral evaluation (Gradshteyn and Ryzhik, 1980)

$$
\frac{1}{R}=\int_{0}^{\infty} J_{0}(\alpha \xi) e^{-\xi z} d \xi
$$

where $\alpha$ and $R$ are defined as

$$
\alpha^{2}=\rho^{2}+\rho_{0}^{2}-2 \rho \rho_{0} \cos \left(\theta-\theta_{0}\right), \quad R^{2}=\alpha^{2}+z^{2} .
$$

Now the Bessel function may be expanded using the addition theorem (Watson, 1980)

$$
J_{0}(\alpha \xi)=\sum_{-\infty}^{\infty} J_{|n|}(\rho \xi) J_{|n|}\left(\rho_{0} \xi\right) e^{i n\left(\theta-\theta_{0}\right)}
$$

Combining the above results leads to the following representation for the reciprocal of the distance between the points $\left(\rho_{0}, \theta_{0}\right)$ on the surface of a half space and the interior point $(\rho, \theta, z)$ as

$$
\frac{1}{R}=\sum_{-\infty}^{\infty} \int_{0}^{\infty} J_{|n|}(\rho \xi) J_{|n|}\left(\rho_{0} \xi\right) e^{-\xi z} d \xi e^{i n\left(\theta-\theta_{0}\right)}
$$


The last step is to multiply the above equation by $\rho_{0} d \rho_{0} d \theta_{0}$ and integrate both sides from $0<\rho_{0}<a, 0<\theta_{0}<2 \pi$. Using orthogonality of the Fourier series and the result

$$
\int_{0}^{a} \xi \rho_{0} J_{0}\left(\xi \rho_{0}\right) d \rho_{0}=a J_{1}(\xi a)
$$

the final expression takes the form

$$
\int_{0}^{2 \pi} \int_{0}^{a} \frac{1}{R} \rho_{0} d \rho_{0} d \theta_{0}=2 \pi a \int_{0}^{\infty} \xi^{-1} J_{1}(a \xi) J_{0}(\rho \xi) e^{-\xi z} d \xi
$$

Introducing the logarithmic potential function $\psi(\rho, z)$ as

$$
\psi(\rho, z)=\int_{0}^{2 \pi} \int_{0}^{a} \ln [R+z] \rho_{0} d \rho_{0} d \theta_{0}
$$

it is easy to verify

$$
\frac{\partial \psi(\rho, z)}{\partial z}=\int_{0}^{2 \pi} \int_{0}^{a} \frac{1}{R} \rho_{0} d \rho_{0} d \theta_{0}=2 \pi a \int_{0}^{\infty} \xi^{-1} J_{1}(a \xi) J_{0}(\rho \xi) e^{-\xi z} d \xi .
$$

The function $\psi(\rho, z)$ is the linear elastic potential function for a uniform normal pressure applied to the surface of an isotropic (Love, 1929) or transversely isotropic (Hanson and Puja, 1996) half space. The various derivatives of $\psi(\rho, z)$ have been recently evaluated in terms of complete elliptic integrals of the first, second, and third kinds by Hanson and Puja (1996). Using these new results and Eq. (9) will allow $I(\mu, \nu ; \lambda)$ to be conveniently evaluated for integers $\mu, \nu$, and $\lambda$.

3. Elliptic integrals and parameters. In this section the complete elliptic integrals of the first, second, and third kinds are defined as well as the parameters that will be used. These elliptic integrals are denoted as $\mathbf{F}(k), \mathbf{E}(k)$, and $\Pi(n, k)$ respectively. They are given in Legendre normal form as (Gradshteyn and Ryzhik, 1980)

$$
\begin{aligned}
\mathbf{F}(k) & =\int_{0}^{1} \frac{d x}{\left(1-x^{2}\right)^{1 / 2}\left(1-k^{2} x^{2}\right)^{1 / 2}}=\int_{0}^{\pi / 2} \frac{d \theta}{\left(1-k^{2} \sin ^{2} \theta\right)^{1 / 2}} \\
\mathbf{E}(k) & =\int_{0}^{1} \frac{\left(1-k^{2} x^{2}\right)^{1 / 2} d x}{\left(1-x^{2}\right)^{1 / 2}}=\int_{0}^{\pi / 2}\left(1-k^{2} \sin ^{2} \theta\right)^{1 / 2} d \theta \\
\Pi(n, k) & =\int_{0}^{1} \frac{d x}{\left(1-n x^{2}\right)\left(1-x^{2}\right)^{1 / 2}\left(1-k^{2} x^{2}\right)^{1 / 2}}=\int_{0}^{\pi / 2} \frac{d \theta}{\left(1-n \sin ^{2} \theta\right)\left(1-k^{2} \sin ^{2} \theta\right)^{1 / 2}}
\end{aligned}
$$

where $k$ is called the modulus, $k^{\prime}=\left(1-k^{2}\right)^{1 / 2}$ is the complementary modulus and $n$ is referred to as the parameter $\left(0<k, k^{\prime}, n<1\right)$.

The evaluation of $I(\mu, \nu ; \lambda)$ will be explicitly written in terms of $a, \rho, z$, and the two parameters $l_{1}(a), l_{2}(a)$ given as

$$
\begin{aligned}
& l_{1}(a)=\frac{1}{2}\left\{\left[(\rho+a)^{2}+z^{2}\right]^{1 / 2}-\left[(\rho-a)^{2}+z^{2}\right]^{1 / 2}\right\}, \\
& l_{2}(a)=\frac{1}{2}\left\{\left[(\rho+a)^{2}+z^{2}\right]^{1 / 2}+\left[(\rho-a)^{2}+z^{2}\right]^{1 / 2}\right\} .
\end{aligned}
$$


These parameters, first introduced by Fabrikant (1989), allow the three-dimensional distance between the point $(a, 0,0)$ on the surface of a half space and the interior point $(\rho, \phi, z)$ to be written in two-dimensional form. That is,

$$
\rho^{2}+a^{2}+z^{2}-2 a \rho \cos (\phi)=l_{1}^{2}(a)+l_{2}^{2}(a)-2 l_{1}(a) l_{2}(a) \cos \phi,
$$

where

$$
l_{1}^{2}(a)+l_{2}^{2}(a)=\rho^{2}+a^{2}+z^{2}, \quad l_{1}(a) l_{2}(a)=a \rho .
$$

Note that it is easy to see $l_{1}(a)<l_{2}(a)$, while it can also be shown that $l_{1}(a)<\rho$. In the solution derived subsequently, the modulus $k$ and the parameter $n$ in Eqs. (10-12) will be given as

$$
k=\frac{l_{1}(a)}{l_{2}(a)}, \quad n=\frac{l_{1}^{2}(a)}{\rho^{2}} .
$$

Under this definition of the modulus and parameter, it can be shown that $0<k^{2}<n<1$. This situation is a circular case, classified as case II by Byrd and Friedman (1971).

4. Forms used by Eason et al. The elliptic integrals used by Eason et al. (1955) are denoted as $\mathbf{F}_{0}\left(k_{s}\right), \mathbf{E}_{0}\left(k_{s}\right)$, and $\Lambda_{0}(\alpha, \beta)$, where $k_{s}$ is the modulus in their paper and $p$ is the parameter. The function $\Lambda_{0}(\alpha, \beta)$ is Heuman's Lambda function and it can appear in the evaluation of the elliptic integral of the third kind for circular cases (Byrd and Friedman, 1971). These elliptic integrals are defined in their paper as

$$
\mathbf{F}_{0}\left(k_{s}\right)=\frac{2}{\pi} \mathbf{F}\left(k_{s}\right), \quad \mathbf{E}_{0}\left(k_{s}\right)=\frac{2}{\pi} \mathbf{E}\left(k_{s}\right), \quad \Lambda_{0}(\alpha, \beta)=\frac{2}{\pi}(1-p)^{1 / 2}\left[1-\frac{k_{s}^{2}}{p}\right]^{1 / 2} \Pi\left(p, k_{s}\right) .
$$

The functions $\mathbf{F}_{0}, \mathbf{E}_{0}$, and $\Lambda_{0}$ were tabulated by Heuman, which apparently motivated their use. The modulus $k_{s}$, parameter $p$, and angle $\beta$ are related by

$$
k_{s}^{2}=\frac{4 a \rho}{(a+\rho)^{2}+z^{2}}, \quad \sin ^{2} \beta=\frac{p-k_{s}^{2}}{p\left(1-k_{s}^{2}\right)}=\frac{z^{2}}{(a-\rho)^{2}+z^{2}} .
$$

Solving this last equation for the parameter $p$ leads to the result

$$
p=\frac{k_{s}^{2}}{1-\left(1-k_{s}^{2}\right) \sin ^{2} \beta}=\frac{4 a \rho}{(a+\rho)^{2}},
$$

and it is easily shown that $0<k_{s}^{2}<p<1$.

In order to compare with the expressions derived in the present paper, the following relations are needed:

$$
k_{s}^{2}=\frac{4 a \rho}{(a+\rho)^{2}+z^{2}}=\frac{4 l_{1}(a) l_{2}(a)}{\left[l_{1}(a)+l_{2}(a)\right]^{2}}, \quad k_{s}=\frac{2 \sqrt{k}}{1+k}, \quad k_{s}^{\prime}=\frac{1-k}{1+k},
$$

where Eq. (15) has been used and $k$ is defined in Eq. (16). Using the above results it is easy to verify that

$$
1-p=\frac{(a-\rho)^{2}}{(a+\rho)^{2}}, \quad 1-\frac{k_{s}^{2}}{p}=\frac{z^{2}}{(a+\rho)^{2}+z^{2}}=\frac{z^{2}}{\left[l_{1}(a)+l_{2}(a)\right]^{2}} .
$$

From Eq. (17), $\Lambda_{0}(\alpha, \beta)$ can now be written as

$$
\Lambda_{0}(\alpha, \beta)=\frac{2}{\pi} \frac{|a-\rho|}{(a+\rho)} \frac{z}{\left[l_{1}(a)+l_{2}(a)\right]} \Pi\left(p, k_{s}\right) .
$$


5. Transformation formulas for complete elliptic integrals. Since the integral evaluations by Eason et al. (1955) involve the quantities $p$ and $k_{s}$ while the present evaluations are in terms of $n$ and $k$, some conversion formulas are necessary in order to verify equivalence. These are provided by Landen's or Gauss' transformation (see Byrd and Friedman, 1971). For the complete elliptic integrals of the first and second kind, the transformations are

$$
\begin{aligned}
& \mathbf{F}_{0}\left(k_{s}\right)=\frac{2}{\pi} \mathbf{F}\left(k_{s}\right)=\frac{2}{\pi} \mathbf{F}\left(\frac{2 \sqrt{k}}{1+k}\right)=\frac{2}{\pi}(1+k) \mathbf{F}(k), \\
& \mathbf{E}_{0}\left(k_{s}\right)=\frac{2}{\pi} \mathbf{E}\left(k_{s}\right)=\frac{2}{\pi} \mathbf{E}\left(\frac{2 \sqrt{k}}{1+k}\right)=\frac{2\left[2 \mathbf{E}(k)-\left(1-k^{2}\right) \mathbf{F}(k)\right]}{\pi(1+k)} .
\end{aligned}
$$

A similar type of relation is needed to convert between $\Pi\left(p, k_{s}\right)$ and $\Pi(n, k)$. This was recently derived by Hanson and Puja (1996) for $z>0$ as

$$
\begin{aligned}
\Pi\left(p, k_{s}\right)= & \Pi\left(p, \frac{2 \sqrt{k}}{1+k}\right)=\frac{\pi l_{2}(a)(1+k)(a+\rho)}{4 z \rho^{2}(a-\rho)}\left\{2 \rho^{2}-\left|a^{2}-\rho^{2}\right|+\frac{a^{4}-\rho^{4}}{\left|a^{2}-\rho^{2}\right|}\right\} \\
& +\frac{(1+k)(a+\rho)}{(a-\rho)}\{\mathbf{F}(k)-2 \Pi(n, k)\} .
\end{aligned}
$$

An additional result that will be required is now obtained. It is shown in the next section that

$$
I(1,1 ;-1)=\frac{a}{2 \rho}+\frac{z l_{2}(a)}{\pi a \rho} \mathbf{E}(k)-\frac{z\left[\rho^{2}+l_{2}^{2}(a)\right]}{\pi a \rho l_{2}(a)} \mathbf{F}(k)+\frac{z\left[\rho^{2}-a^{2}\right]}{\pi a \rho l_{2}(a)} \Pi(n, k) .
$$

Since both Bessel functions have the same order, the result should be identical with $a$ and $\rho$ reversed. If in the above equation these variables are interchanged (where $l_{1}(a), l_{2}(a)$, and $k$ are unaltered by this transformation) and the new equation is set equal to the one above, the result is

$$
\Pi\left(p_{1}, k\right)=\frac{\pi l_{2}(a)}{2 z}+\mathbf{F}(k)-\Pi(n, k), \quad p_{1}=\frac{l_{1}^{2}(a)}{a^{2}}=\frac{\rho^{2}}{l_{2}^{2}(a)}=\frac{k^{2}}{n} .
$$

This formula can be written in general form as

$$
\Pi\left(\frac{k^{2}}{n}, k\right)=\frac{\pi}{2}\left[\frac{n}{(1-n)\left(n-k^{2}\right)}\right]^{1 / 2}+\mathbf{F}(k)-\Pi(n, k),
$$

which agrees with a result given in Byrd and Friedman (1971). In the present analysis, Eq. (26) will be utilized since it is a simplification of the above equation to the present definitions of $k$ and $n$.

There are several other transformation formulas in Byrd and Friedman (1971) for the elliptic integral of the third kind. Although these formulas might lead to Eq. (24) above, the author was not able to demonstrate this in a simple fashion. 
6. Differential formulas for elliptic integrals. In this section some differential formulas for complete elliptic integrals of all three kinds are presented or derived. From Eqs. (10-12) these integrals depend on the parameters $k$ and $n$. These two parameters are functions of $\rho, a$, and $z$ through Eq. (16). To differentiate the complete elliptic integrals with respect to $\rho, a$, or $z$, the chain rule may be used. The necessary derivatives needed for the complete elliptic integrals of the first and second kinds are given by Gradshteyn and Ryzhik (1980) as

$$
\frac{\partial \mathbf{E}(k)}{\partial k}=\frac{\mathbf{E}(k)-\mathbf{F}(k)}{k}, \quad \frac{\partial \mathbf{F}(k)}{\partial k}=-\frac{\mathbf{F}(k)}{k}+\frac{\mathbf{E}(k)}{k\left(1-k^{2}\right)} .
$$

Additionally, one can verify the results

$$
\frac{\partial k}{\partial z}=-\frac{2 z k}{l_{2}^{2}(a)\left(1-k^{2}\right)}, \quad \frac{\partial k}{\partial \rho}=\frac{a\left(a^{2}+z^{2}-\rho^{2}\right)}{l_{2}^{4}(a)\left(1-k^{2}\right)}, \quad \frac{\partial k}{\partial a}=\frac{\rho\left(\rho^{2}+z^{2}-a^{2}\right)}{l_{2}^{4}(a)\left(1-k^{2}\right)},
$$

where we have used the following differential relations provided by Fabrikant (1989):

$$
\begin{aligned}
\frac{\partial l_{1}(a)}{\partial z} & =-\frac{z l_{1}(a)}{l_{2}^{2}(a)-l_{1}^{2}(a)}, & \frac{\partial l_{2}(a)}{\partial z} & =\frac{z l_{2}(a)}{l_{2}^{2}(a)-l_{1}^{2}(a)}, \\
\frac{\partial l_{1}(a)}{\partial \rho} & =\frac{\rho\left[a^{2}-l_{1}^{2}(a)\right]}{l_{1}(a)\left[l_{2}^{2}(a)-l_{1}^{2}(a)\right]}, & \frac{\partial l_{2}(a)}{\partial \rho} & =\frac{\rho\left[l_{2}^{2}(a)-a^{2}\right]}{l_{2}(a)\left[l_{2}^{2}(a)-l_{1}^{2}(a)\right]}, \\
\frac{\partial l_{1}(a)}{\partial a} & =\frac{a\left[\rho^{2}-l_{1}^{2}(a)\right]}{l_{1}(a)\left[l_{2}^{2}(a)-l_{1}^{2}(a)\right]}, & \frac{\partial l_{2}(a)}{\partial a} & =\frac{a\left[l_{2}^{2}(a)-\rho^{2}\right]}{l_{2}(a)\left[l_{2}^{2}(a)-l_{1}^{2}(a)\right]} .
\end{aligned}
$$

It will also be necessary to differentiate the complete elliptic integral of the third kind with respect to the variables $\rho, a$, or $z$. The derivatives with respect to $k$ and $n$ are given by Byrd and Friedman (1971) as

$$
\begin{aligned}
& \frac{\partial \Pi(n, k)}{\partial k}=\frac{k}{\left(n-k^{2}\right)}\left[\Pi(n, k)-\frac{1}{\left(1-k^{2}\right)} \mathbf{E}(k)\right], \\
& \frac{\partial \Pi(n, k)}{\partial n}=\frac{1}{2 n(1-n)\left(n-k^{2}\right)}\left[n \mathbf{E}(k)-\left(n-k^{2}\right) \mathbf{F}(k)+\left(n^{2}-k^{2}\right) \Pi(n, k)\right] .
\end{aligned}
$$

The last results needed for application of the chain rule are the derivatives of $n$. These are

$$
\frac{\partial n}{\partial z}=-\frac{2 z k^{2}}{\rho^{2}\left(1-k^{2}\right)}, \quad \frac{\partial n}{\partial \rho}=-\frac{2 k^{2}\left[\rho^{2}-l_{1}^{2}(a)\right]}{\rho^{3}\left(1-k^{2}\right)}, \quad \frac{\partial n}{\partial a}=\frac{2 k\left[\rho^{2}-l_{1}^{2}(a)\right]}{\rho^{3}\left(1-k^{2}\right)} .
$$

Putting these equations together, the derivatives of $\Pi(n, k)$ become

$$
\begin{aligned}
\frac{\partial \Pi(n, k)}{\partial z} & =\frac{\partial \Pi(n, k)}{\partial n} \frac{\partial n}{\partial z}+\frac{\partial \Pi(n, k)}{\partial k} \frac{\partial k}{\partial z} \\
& =\frac{\left[\rho^{2}-z^{2}-a^{2}\right]}{z l_{2}^{2}(a)\left(1-k^{2}\right)^{2}} \mathbf{E}(k)+\frac{\left[l_{2}^{2}(a)-\rho^{2}\right]}{z l_{2}^{2}(a)\left(1-k^{2}\right)} \mathbf{F}(k)+\frac{\left[z^{2}+l_{1}^{2}(a)-l_{2}^{2}(a)\right]}{z l_{2}^{2}(a)\left(1-k^{2}\right)} \Pi(n, k) \\
\frac{\partial \Pi(n, k)}{\partial \rho} & =\frac{\partial \Pi(n, k)}{\partial n} \frac{\partial n}{\partial \rho}+\frac{\partial \Pi(n, k)}{\partial k} \frac{\partial k}{\partial \rho} \\
& =-\frac{2 \rho}{l_{2}^{2}(a)\left(1-k^{2}\right)^{2}} \mathbf{E}(k)+\frac{\rho}{l_{2}^{2}(a)\left(1-k^{2}\right)} \mathbf{F}(k)+\frac{\rho\left[l_{2}^{2}(a)-a^{2}\right]}{l_{2}^{4}(a)\left(1-k^{2}\right)} \Pi(n, k)
\end{aligned}
$$


7. Evaluations for integers less than 2. Now the integral in Eq. (1) is evaluated when $\mu, \nu$, and $\lambda$ take on the values $-1,0$, and 1 . Without loss of generality, $\mu$ and $\nu$ are taken as 0 or 1 since $J_{-n}(x)=(-1)^{n} J_{n}(x)$. Since $\mu$ and $\nu$ can take on two different values and $\lambda$ three, there are a total of twelve different combinations. However, the integral $I(0,0 ;-1)$ is nonconvergent and not presently considered leaving eleven possibilities. Many of these integrals will be expressed in terms of derivatives of the potential function $\psi(\rho, z)$ defined in Eq. (8). These derivatives have been evaluated by Hanson and Puja (1996) and the necessary results are taken from there. The remaining evaluations will be found by using the relations above.

As a starting point, Eq. (9) provides

$$
I(1,0 ;-1)=\frac{1}{2 \pi a} \frac{\partial \psi(\rho, z)}{\partial z}=\frac{2}{\pi a l_{2}(a)}\left[l_{2}^{2}(a) \mathbf{E}(k)-\left[\rho^{2}-l_{1}^{2}(a)\right] \mathbf{F}(k)-z^{2} \Pi(n, k)\right] .
$$

Applying a $\rho$ derivative to this equation leads to

$$
I(1,1 ; 0)=-\frac{1}{2 \pi a} \frac{\partial^{2} \psi(\rho, z)}{\partial \rho \partial z}=\frac{2 l_{2}(a)}{\pi a \rho}[\mathbf{F}(k)-\mathbf{E}(k)] .
$$

Integrating the above equation with respect to $z$ yields

$$
\begin{aligned}
I(1,1 ;-1)=\frac{1}{2 \pi a} \frac{\partial \psi(\rho, z)}{\partial \rho}= & \frac{a}{2 \rho} \\
& +\frac{z l_{2}(a)}{\pi a \rho} \mathbf{E}(k) \\
& -\frac{z}{\pi a \rho l_{2}(a)}\left[\rho^{2}+l_{2}^{2}(a)\right] \mathbf{F}(k)-\frac{z\left(a^{2}-\rho^{2}\right)}{\pi a \rho l_{2}(a)} \Pi(n, k) .
\end{aligned}
$$

In performing this integration an arbitrary function of $\rho$ and $a$ may be added. It can be shown that this function is zero by considering $z \rightarrow \infty$.

Three more evaluations can be obtained by taking $z$ derivatives as follows. Applying a $z$ derivative to Eq. (37) leads to

$$
I(1,1 ; 1)=\frac{1}{2 \pi a} \frac{\partial^{3} \psi(\rho, z)}{\partial \rho \partial z^{2}}=\frac{2 z}{\pi a \rho l_{2}(a)\left(1-k^{2}\right)}\left[-\mathbf{F}(k)+\frac{1+k^{2}}{1-k^{2}} \mathbf{E}(k)\right] .
$$

Applying a $z$ derivative to Eq. (36) results in

$$
I(1,0 ; 0)=-\frac{1}{2 \pi a} \frac{\partial^{2} \psi(\rho, z)}{\partial z^{2}}=-\frac{2 z}{\pi a l_{2}(a)}[\mathbf{F}(k)-\Pi(n, k)]
$$

A $z$ derivative to the above equation gives

$$
I(1,0 ; 1)=\frac{1}{2 \pi a} \frac{\partial^{3} \psi(\rho, z)}{\partial z^{3}}=\frac{2\left[l_{2}^{2}(a)-a^{2}\right]}{\pi a l_{2}^{3}(a)\left(1-k^{2}\right)} \mathbf{F}(k)-\frac{2\left[z^{2}+\rho^{2}-a^{2}\right]}{\pi a l_{2}^{3}(a)\left(1-k^{2}\right)} \mathbf{E}(k) .
$$

Three more relations can be found by interchanging the variables $a$ and $\rho$ in some of the above expressions and using the identity in Eq. (26). Switching these in the expression for $I(1,0 ;-1)$ provides

$$
I(0,1 ;-1)=\frac{2}{\pi \rho l_{2}(a)}\left[-\frac{z \pi l_{2}(a)}{2}+l_{2}^{2}(a) \mathbf{E}(k)-\left[l_{2}^{2}(a)-\rho^{2}\right] \mathbf{F}(k)+z^{2} \Pi(n, k)\right] .
$$


A similar procedure to $I(1,0 ; 0)$ and $I(1,0 ; 1)$ leads to

$$
\begin{gathered}
I(0,1 ; 0)=\frac{1}{\rho}\left[1-\frac{2 z}{\pi l_{2}(a)} \Pi(n, k)\right], \\
I(0,1 ; 1)=\frac{2}{\pi \rho l_{2}^{3}(a)\left(1-k^{2}\right)}\left[\left[l_{2}^{2}(a)-\rho^{2}\right] \mathbf{F}(k)-\frac{\left[z^{2}+a^{2}-\rho^{2}\right]}{\left(1-k^{2}\right)} \mathbf{E}(k)\right] .
\end{gathered}
$$

The remaining two results are obtained as follows. For $I(0,0 ; 0)$, one can write

$$
I(0,0 ; 0)=\frac{1}{\rho} \frac{\partial}{\partial \rho}[\rho I(0,1 ;-1)] .
$$

Substituting for $I(0,1 ;-1)$ from above and performing the derivative using the results in Sec. 6 gives

$$
I(0,0 ; 0)=\frac{2}{\pi l_{2}(a)} \mathbf{F}(k) .
$$

Differentiating this equation with respect to $z$ yields

$$
I(0,0 ; 1)=\frac{2 z}{\pi l_{2}^{3}(a)\left(1-k^{2}\right)}\left[-\mathbf{F}(k)+\frac{2}{1-k^{2}} \mathbf{E}(k)\right] .
$$

8. Comparison with Eason et al. A comparison is now made with the results of Eason et al. (1955). For the integral evaluations containing only complete elliptic integrals of the first and second kind, the comparisons are easily made. For example consider $I(0,0 ; 0)$. Eason's result can be transformed using Eqs. $(15,20,23)$ as follows:

$$
I(0,0 ; 0)=\frac{k_{s}}{2(a \rho)^{1 / 2}} \mathbf{F}_{0}\left(k_{s}\right)=\frac{2 \sqrt{k}}{1+k} \frac{1}{2\left[l_{1}(a) l_{2}(a)\right]^{1 / 2}} \frac{2}{\pi}(1+k) \mathbf{F}(k)=\frac{2}{\pi l_{2}(a)} \mathbf{F}(k),
$$

which agrees with the present result in Eq. (46). The other evaluations not involving the complete elliptic integral of the third kind can be shown to be equivalent in a similar fashion.

The integral evaluations by Eason et al. (1955), which require the elliptic integral of the third kind, are in a slightly more complicated form. The complication is due to the different expressions required in the regions $\rho<a$ and $\rho>a$. To illustrate a comparison $I(1,0 ; 0)$ will be used. Starting with Eq. (40) and substituting for $\Pi(n, k)$ in terms of $\Pi\left(p, k_{s}\right)$ through Eq. (24) leads to

$$
\begin{aligned}
I(1,0 ; 0)=\frac{1}{4 a \rho^{2}}\left\{2 \rho^{2}-\left|a^{2}-\rho^{2}\right|+\frac{a^{4}-\rho^{4}}{\left|a^{2}-\rho^{2}\right|}\right\} & -\frac{z}{\pi a l_{2}(a)} \mathbf{F}(k) \\
& -\frac{z(a-\rho)}{\pi a l_{2}(a)(a+\rho)(1+k)} \Pi\left(p, k_{s}\right) .
\end{aligned}
$$

Now using Eqs. $(22,23)$ and the result $(1+k) l_{2}(a)=2(a \rho)^{1 / 2} / k_{s}$ provides

$$
I(1,0 ; 0)=\frac{1}{4 a \rho^{2}}\left\{2 \rho^{2}-\left|a^{2}-\rho^{2}\right|+\frac{a^{4}-\rho^{4}}{\left|a^{2}-\rho^{2}\right|}\right\}-\frac{z k_{s}}{4 a(a \rho)^{1 / 2}} \mathbf{F}_{0}\left(k_{s}\right)-\frac{(a-\rho)}{2 a|a-\rho|} \Lambda_{0}(\alpha, \beta) .
$$

This agrees with the evaluation in Eason et al. (1955) for $\rho<a$ and $\rho>a$. The above equation indicates that the results in Eason et al. (1955) can be written as a single expression for all $\rho, a$. However, the present form as in Eq. (40) appears to be simpler. The remaining expressions can be shown to be equivalent in a similar manner. 
9. A result derived by Sura-Bura. It was pointed out by Eason et al. (1955) that the integral $I(1,0 ; 0)$ was also evaluated by Sura-Bura (1950). Although the present authors have not seen this paper, it appears that sufficient details are provided by Eason et al. (1955). From their paper (p. 541) the result is

$$
I(1,0 ; 0)=\frac{1}{a}\left[1-\Lambda_{0}\left(k_{1}, \beta_{1}\right)\right]
$$

with

$$
\begin{aligned}
k_{1}^{2}= & \frac{x_{2}\left(x_{1}-2 z^{2}\right)}{x_{1}\left(x_{2}+2 z^{2}\right)}, \quad \sin ^{2} \beta_{1}=\frac{x_{1}}{x_{1}+x_{2}}, \quad x_{1}=W-a^{2}+\rho^{2}+z^{2}, \\
& x_{2}=W+a^{2}-\rho^{2}-z^{2}, \quad W^{2}=\left(a^{2}+\rho^{2}+z^{2}\right)^{2}-4 a^{2} \rho^{2},
\end{aligned}
$$

where what appears to be a misprint in the denominator of the equation defining $k_{1}^{2}$ has been presently corrected. It is easy to verify directly that

$$
W=l_{2}^{2}(a)-l_{1}^{2}(a), \quad x_{1}=2\left[l_{2}^{2}(a)-a^{2}\right], \quad x_{2}=2\left[a^{2}-l_{1}^{2}(a)\right],
$$

leading to the results

$$
k_{1}^{2}=\frac{\left[a^{2}-l_{1}^{2}(a)\right]\left[\rho^{2}-l_{1}^{2}(a)\right]}{\left[l_{2}^{2}(a)-a^{2}\right]\left[l_{2}^{2}(a)-\rho^{2}\right]}=\frac{z^{2} l_{1}^{2}(a)}{z^{2} l_{2}^{2}(a)}=k^{2}, \quad \sin ^{2} \beta_{1}=\frac{l_{2}^{2}(a)-a^{2}}{l_{2}^{2}(a)-l_{1}^{2}(a)},
$$

and the modulus used by Sura-Bura is identical to the one used presently. If a new variable $p_{1}$ is defined to be consistent with Eq. (19) as

$$
p_{1}=\frac{k_{1}^{2}}{1-\left(1-k_{1}^{2}\right) \sin ^{2} \beta_{1}}, \quad 1-\left(1-k_{1}^{2}\right) \sin ^{2} \beta_{1}=\frac{a^{2}}{l_{2}^{2}(a)}=\frac{l_{1}^{2}(a)}{\rho^{2}},
$$

then it is easy to show that $p_{1}$ is as defined in Eq. (26).

To arrive at the final form of Eq. (51), the function $\Lambda_{0}\left(k_{1}, \beta_{1}\right)$ can be written as

$$
\Lambda_{0}\left(k_{1}, \beta_{1}\right)=\frac{2}{\pi}\left(1-p_{1}\right)^{1 / 2}\left[1-\frac{k_{1}^{2}}{p_{1}}\right]^{1 / 2} \Pi\left(p_{1}, k_{1}\right)=\frac{2 z}{\pi l_{2}(a)} \Pi\left(p_{1}, k\right),
$$

where $k_{1}=k$ has been used along with the identity from Fabrikant (1989):

$$
\left[l_{2}^{2}(a)-\rho^{2}\right]\left[\rho^{2}-l_{1}^{2}(a)\right]=z^{2} \rho^{2} .
$$

In the present notation, Sura-Bura's formula in Eq. (51) becomes

$$
I(1,0 ; 0)=\frac{1}{a}\left[1-\frac{2 z}{\pi l_{2}(a)} \Pi\left(p_{1}, k\right)\right]
$$

Application of Eq. (26) leads finally to Eq. (40) above. 
10. Evaluations when $\mu$ or $\nu$ may be 2. In this section the integral in Eq. (1) is evaluated when either $\mu$ or $\nu$ or both take on the value 2 . The parameter $\lambda$ is still $-1,0$, or 1 . As in Sec. 7 , some of these integrals can be written as derivatives of the potential function $\psi(\rho, z)$ defined in Eq. (8). Also, the potential function $\chi(\rho, z)$ which is the integral of $\psi(\rho, z)$ with respect to $z$ will be used. The function $\chi(\rho, z)$ is the linear elastic potential function for uniform shear loading over a circular area on a half space. Various derivatives of both $\psi(\rho, z)$ and $\chi(\rho, z)$ are tabulated in Hanson and Puja (1996).

The evaluation of two integrals can be written down directly in terms of $\psi(\rho, z)$ as

$$
\begin{aligned}
I(1,2 ; 0) & =-\frac{1}{2 \pi a} e^{-2 i \phi} \Lambda^{2} \psi(\rho, z) \\
& =\frac{a}{\rho^{2}}+\frac{2 z l_{2}(a)}{\pi a \rho^{2}} \mathbf{E}(k)-\frac{2 z l_{2}(a)}{\pi a \rho^{2}} \mathbf{F}(k)-\frac{2 z a}{\pi \rho^{2} l_{2}(a)} \Pi(n, k), \\
I(1,2 ; 1) & =\frac{1}{2 \pi a} e^{-2 i \phi} \frac{\partial}{\partial z} \Lambda^{2} \psi(\rho, z) \\
& =\frac{2\left[2 l_{2}^{2}(a)-l_{1}^{2}(a)-\rho^{2}\right]}{\pi a \rho^{2} l_{2}(a)\left(1-k^{2}\right)} \mathbf{F}(k)+\frac{2\left[\rho^{2}\left(z^{2}+\rho^{2}-a^{2}\right)-2\left(l_{2}^{2}(a)-l_{1}^{2}(a)\right)^{2}\right]}{\pi a \rho^{2} l_{2}^{3}(a)\left(1-k^{2}\right)^{2}} \mathbf{E}(k),
\end{aligned}
$$

where the differential operator $\Lambda^{2}$ is defined in Hanson and Puja (1996). Integrating Eq. (59) with respect to $z$ (and noting that the arbitrary function vanishes) also provides

$$
\begin{aligned}
I(1,2 ;-1)= & \frac{1}{2 \pi a} e^{-2 i \phi} \Lambda^{2} \chi(\rho, z) \\
= & -\frac{z a}{\rho^{2}}+\frac{2 l_{2}(a)\left[2 a^{2}-\rho^{2}-z^{2}\right]}{3 \pi a \rho^{2}} \mathbf{E}(k)+\frac{2 l_{2}(a)\left[l_{1}^{2}(a)-2 a^{2}+\rho^{2}+z^{2}\right]}{3 \pi a \rho^{2}} \mathbf{F}(k) \\
& +\frac{2 a z^{2}}{\pi \rho^{2} l_{2}(a)} \Pi(n, k) .
\end{aligned}
$$

Interchanging $a$ and $\rho$ in the above equations and using Eq. (26) leads to

$$
\begin{aligned}
I(2,1 ; 0)= & \frac{2 z l_{2}(a)}{\pi a^{2} \rho} \mathbf{E}(k)-\frac{2 z\left[\rho^{2}+l_{2}^{2}(a)\right]}{\pi a^{2} \rho l_{2}(a)} \mathbf{F}(k)+\frac{2 z \rho}{\pi a^{2} l_{2}(a)} \Pi(n, k), \\
I(2,1 ; 1)= & \frac{2\left[2 l_{2}^{2}(a)-l_{1}^{2}(a)-a^{2}\right]}{\pi a^{2} \rho l_{2}(a)\left(1-k^{2}\right)} \mathbf{F}(k) \\
& \quad+\frac{2\left[a^{2}\left(z^{2}+a^{2}-\rho^{2}\right)-2\left(l_{2}^{2}(a)-l_{1}^{2}(a)\right)^{2}\right]}{\pi a^{2} \rho l_{2}^{3}(a)\left(1-k^{2}\right)^{2}} \mathbf{E}(k) . \\
I(2,1 ;-1)= & \frac{2 l_{2}(a)\left[2 \rho^{2}-a^{2}-z^{2}\right]}{3 \pi a^{2} \rho} \mathbf{E}(k) \\
& +\frac{2\left\{l_{2}^{2}(a)\left[l_{2}^{2}(a)-l_{1}^{2}(a)\right]-3 \rho^{2}\left[\rho^{2}-l_{1}^{2}(a)\right]\right\}}{3 \pi a^{2} \rho l_{2}(a)} \mathbf{F}(k) \\
& -\frac{2 \rho z^{2}}{\pi a^{2} l_{2}(a)} \Pi(n, k) .
\end{aligned}
$$

Additional results can be obtained from the recurrence relation (Eason et al., 1955)

$$
2 \nu I(\mu, \nu ; \lambda)=\rho I(\mu, \nu+1 ; \lambda+1)+\rho I(\mu, \nu-1 ; \lambda+1) .
$$


Setting $\mu=0, \nu=1$, and $\lambda=0$ leads to

$$
I(0,2 ; 1)=\frac{2}{\rho^{2}}+\frac{2 z}{\pi l_{2}^{3}(a)\left(1-k^{2}\right)}\left[\mathbf{F}(k)-\frac{2}{1-k^{2}} \mathbf{E}(k)\right]-\frac{4 z}{\pi \rho^{2} l_{2}(a)} \Pi(n, k),
$$

while setting $\mu=0, \nu=1$, and $\lambda=-1$ provides

$$
I(0,2 ; 0)=\frac{2}{\pi \rho^{2} l_{2}(a)}\left[-z \pi l_{2}(a)+2 l_{2}^{2}(a) \mathbf{E}(k)+\left[\rho^{2}-2 l_{2}^{2}(a)\right] \mathbf{F}(k)+2 z^{2} \Pi(n, k)\right] .
$$

Interchanging $a$ and $\rho$ in the last two expressions and using Eq. (26) also gives

$$
\begin{aligned}
& I(2,0 ; 1)=-\frac{4 z}{\pi l_{2}^{3}(a)\left(1-k^{2}\right)^{2}} \mathbf{E}(k)+\frac{2 z\left[a^{2}+2 l_{1}^{2}(a)-2 l_{2}^{2}(a)\right]}{\pi a^{2} l_{2}^{3}(a)\left(1-k^{2}\right)} \mathbf{F}(k)+\frac{4 z}{\pi a^{2} l_{2}(a)} \Pi(n, k), \\
& I(2,0 ; 0)=\frac{2}{\pi a^{2} l_{2}(a)}\left[2 l_{2}^{2}(a) \mathbf{E}(k)+\left[a^{2}+2 z^{2}-2 l_{2}^{2}(a)\right] \mathbf{F}(k)-2 z^{2} \Pi(n, k)\right] .
\end{aligned}
$$

The last two integrals obtained from Eq. (65) are found with $\mu=2, \nu=1, \lambda=-1$ and $\mu=2, \nu=1$, and $\lambda=0$. The results are

$$
\begin{aligned}
& I(2,2 ; 0)=-\frac{4 l_{2}(a)\left[\rho^{2}+a^{2}+z^{2}\right]}{3 \pi a^{2} \rho^{2}} \mathbf{E}(k)+\frac{2 l_{2}(a)\left[2 l_{2}^{2}(a)+l_{1}^{2}(a)\right]}{3 \pi a^{2} \rho^{2}} \mathbf{F}(k), \\
& I(2,2 ; 1)=\frac{4 z l_{2}(a)\left[1-k^{2}+k^{4}\right]}{\pi a^{2} \rho^{2}\left(1-k^{2}\right)^{2}} \mathbf{E}(k)-\frac{2 z l_{2}(a)\left[2-k^{2}\right]}{\pi a^{2} \rho^{2}\left(1-k^{2}\right)} \mathbf{F}(k) .
\end{aligned}
$$

Of the three remaining integrals, two can be evaluated using recurrence relations from Eason et al. (1955). They are

$$
\begin{aligned}
I(2,2 ;-1)= & \frac{a}{4} I(1,2 ; 0)+\frac{\rho}{4} I(2,1 ; 0)-\frac{z}{4} I(2,2 ; 0) \\
= & \frac{a^{2}}{4 \rho^{2}}+\frac{z l_{2}(a)\left[5 a^{2}+5 \rho^{2}+2 z^{2}\right]}{6 \pi a^{2} \rho^{2}} \mathbf{E}(k) \\
& \quad-\frac{z\left\{l_{2}^{2}(a)\left[4 a^{2}+4 \rho^{2}+z^{2}+l_{2}^{2}(a)\right]+3 \rho^{4}\right\}}{6 \pi a^{2} \rho^{2} l_{2}(a)} \mathbf{F}(k) \\
& \quad+\frac{z\left[\rho^{4}-a^{4}\right]}{2 \pi a^{2} \rho^{2} l_{2}(a)} \Pi(n, k), \\
I(2,0 ;-1)= & \frac{a}{2} I(1,0 ; 0)-\frac{\rho}{2} I(2,1 ; 0)-\frac{z}{2} I(2,0 ; 0) \\
= & -\frac{3 z l_{2}(a)}{\pi a^{2}} \mathbf{E}(k)+\frac{z\left[3 l_{2}^{2}(a)+\rho^{2}-2 z^{2}-2 a^{2}\right\}}{\pi a^{2} l_{2}(a)} \mathbf{F}(k) \\
& +\frac{z\left[a^{2}-\rho^{2}+2 z^{2}\right]}{\pi a^{2} l_{2}(a)} \Pi(n, k) .
\end{aligned}
$$

Interchanging $a$ and $\rho$ in the last expression and using Eq. (26) gives the final result:

$$
\begin{aligned}
I(0,2 ;-1)= & \frac{\left[\rho^{2}-a^{2}+2 z^{2}\right]}{2 \rho^{2}}-\frac{3 z l_{2}(a)}{\pi \rho^{2}} \mathbf{E}(k) \\
& +\frac{z\left\{3 l_{2}^{2}(a)-\rho^{2}\right\}}{\pi \rho^{2} l_{2}(a)} \mathbf{F}(k)-\frac{z\left[\rho^{2}-a^{2}+2 z^{2}\right]}{\pi \rho^{2} l_{2}(a)} \Pi(n, k) .
\end{aligned}
$$


11. Evaluations when $\lambda=-2,2$. The remaining integrals to be evaluated are when the parameter $\lambda$ takes on the values -2 or 2 while $\mu$ or $\nu$ are less than or equal to 2 . There are 18 integrals in this category. However, the three integrals $I(0,0 ;-2), I(1,0 ;-2)$, and $I(0,1 ;-2)$ are nonconvergent. The 15 convergent integrals can be evaluated by differentiation of the above results or by the recurrence relations in Eason et al. (1955). Further details are omitted and the results are now given as

$$
\begin{aligned}
I(1,1 ;-2)= & -\frac{z a}{2 \rho}+\frac{l_{2}(a)\left[2 \rho^{2}+2 a^{2}-z^{2}\right]}{3 \pi a \rho} \mathbf{E}(k) \\
& +\frac{l_{2}^{2}(a)\left[4 l_{1}^{2}(a)+z^{2}-2 \rho^{2}-2 a^{2}\right]+3 z^{2} \rho^{2}}{3 \pi a \rho l_{2}(a)} \mathbf{F}(k) \\
& +\frac{z^{2}\left[a^{2}-\rho^{2}\right]}{\pi a \rho l_{2}(a)} \Pi(n, k), \\
I(2,0 ;-2)= & \frac{l_{2}(a)\left[11 z^{2}+8 a^{2}-4 \rho^{2}\right]}{9 \pi a^{2}} \mathbf{E}(k) \\
& -\frac{\left\{l_{2}^{2}(a)\left[2 l_{2}^{2}(a)+3 z^{2}\right]-6 l_{1}^{2}(a)\left[a^{2}+z^{2}\right]+\rho^{2}\left[6 l_{1}^{2}(a)+4 a^{2}+9 z^{2}-6 \rho^{2}\right]\right\}}{9 \pi a^{2} l_{2}(a)} \mathbf{F}(k) \\
& +\frac{z^{2}\left[3 \rho^{2}-3 a^{2}-2 z^{2}\right]}{3 \pi a^{2} l_{2}(a)} \Pi(n, k), \\
I(0,2 ;-2)= & \frac{z\left[3 a^{2}-3 \rho^{2}-2 z^{2}\right]}{6 \rho^{2}}+\frac{l_{2}(a)\left[11 z^{2}+8 \rho^{2}-4 a^{2}\right]}{9 \pi \rho^{2}} \mathbf{E}(k) \\
& +\frac{\left\{a^{2}\left[6 a^{2}-6 l_{1}^{2}(a)-4 \rho^{2}+6 z^{2}\right]+6 \rho^{2} l_{1}^{2}(a)-2 l_{2}^{4}(a)-3 z^{2}\left[\rho^{2}+3 l_{2}^{2}(a)\right]\right\}}{9 \pi \rho^{2} l_{2}(a)} \mathbf{F}(k) \\
& -\frac{z^{2}\left[3 a^{2}-3 \rho^{2}-2 z^{2}\right]}{3 \pi \rho^{2} l_{2}(a)} \Pi(n, k), \\
& +\frac{z\left\{2 a^{2} \rho^{2}+4 \rho^{2} z^{2}-a^{4}-\rho^{4}\right\}}{4 \pi a^{2} \rho l_{2}(a)} \Pi(n, k) \\
I(2,1 ;-2)= & \frac{a^{2}}{8 \rho}+\frac{z l_{2}(a)\left[5 a^{2}-13 \rho^{2}+2 z^{2}\right]}{12 \pi a^{2} \rho} \mathbf{E}(k) \\
& -\frac{z\left\{a^{2}\left[2 \rho^{2}+4 z^{2}-a^{2}\right]-\rho^{4}\right\}}{4 \pi a \rho^{2} l_{2}(a)} \Pi(n, k) \\
I(1,2 ;-2)= & \frac{a\left[2 \rho^{2}+4 z^{2}-a^{2}\right]}{8 \rho^{2}}+\frac{z\left[2 z^{2}+5 \rho^{2}-13 a^{2}\right]}{12 \pi \rho l_{1}(a)} \mathbf{E}(k) \\
& \frac{z\left\{l_{2}^{2}(a)\left[13 a^{2}-5 l_{1}^{2}(a)-5 \rho^{2}-2 z^{2}\right]-3 \rho^{4}\right\}}{\left.\mathbf{F}(k)-11 a^{2} \rho^{2}-12 z^{2} \rho^{2}-5 a^{2} l_{2}^{2}(a)-2 z^{2} l_{2}^{2}(a)\right\}} \mathbf{F}(k) \\
& \\
&
\end{aligned}
$$




$$
\begin{aligned}
& I(2,2 ;-2)=-\frac{z a^{2}}{4 \rho^{2}} \\
& +\frac{l_{2}(a)\left\{\left[l_{1}^{2}(a)+l_{2}^{2}(a)\right]\left[8 a^{2}+8 \rho^{2}-2 z^{2}\right]-3\left[8 a^{2} \rho^{2}+5 z^{2} \rho^{2}+5 a^{2} z^{2}\right]\right\}}{30 \pi a^{2} \rho^{2}} \mathbf{E}(k) \\
& +\left\{\frac{4 l_{2}^{2}(a)\left[a^{2} l_{1}^{2}(a)-2 a^{4}+2 a^{2} z^{2}+2 \rho^{2} l_{2}^{2}(a)-\rho^{4}\right]}{30 \pi a^{2} \rho^{2} l_{2}(a)}\right. \\
& \left.+\frac{z^{2} l_{2}^{2}(a)\left[z^{2}+l_{2}^{2}(a)\right]+3 \rho^{4}\left[z^{2}+4 l_{1}^{2}(a)-4 \rho^{2}\right]}{30 \pi a^{2} \rho^{2} l_{2}(a)}\right\} \mathbf{F}(k) \\
& +\frac{z^{2}\left[a^{4}-\rho^{4}\right]}{2 \pi a^{2} \rho^{2} l_{2}(a)} \Pi(n, k) \\
& I(0,0 ; 2)=\frac{2\left\{l_{2}^{2}(a)\left(1-k^{2}\right)^{2}-5 z^{2}-3 z^{2} k^{2}\right\}}{\pi l_{2}^{5}(a)\left(1-k^{2}\right)^{3}} \mathbf{F}(k)-\frac{4\left\{l_{2}^{2}(a)\left(1-k^{2}\right)^{2}-4 z^{2}\left(1+k^{2}\right)\right\}}{\pi l_{2}^{5}(a)\left(1-k^{2}\right)^{4}} \mathbf{E}(k), \\
& I(1,0 ; 2)=\frac{2 z\left\{l_{2}^{2}(a)+7 l_{1}^{2}(a)-5 a^{2}-3 a^{2} k^{2}\right\}}{\pi a l_{2}^{5}(a)\left(1-k^{2}\right)^{3}} \mathbf{F}(k) \\
& +\frac{2 z\left\{8 a^{2}\left(1+k^{2}\right)-k^{2} l_{1}^{2}(a)-l_{2}^{2}(a)-14 l_{1}^{2}(a)\right\}}{\pi a l_{2}^{5}(a)\left(1-k^{2}\right)^{4}} \mathbf{E}(k), \\
& I(0,1 ; 2)=\frac{2 z\left\{l_{2}^{2}(a)+7 l_{1}^{2}(a)-5 \rho^{2}-3 \rho^{2} k^{2}\right\}}{\pi \rho l_{2}^{5}(a)\left(1-k^{2}\right)^{3}} \mathbf{F}(k) \\
& +\frac{2 z\left\{8 \rho^{2}\left(1+k^{2}\right)-k^{2} l_{1}^{2}(a)-l_{2}^{2}(a)-14 l_{1}^{2}(a)\right\}}{\pi \rho l_{2}^{5}(a)\left(1-k^{2}\right)^{4}} \mathbf{E}(k) \\
& I(1,1 ; 2)=-\frac{2\left\{-l_{2}^{2}(a)\left(1-k^{2}\right)^{2}+z^{2}+7 z^{2} k^{2}\right\}}{\pi a \rho l_{2}^{3}(a)\left(1-k^{2}\right)^{3}} \mathbf{F}(k) \\
& -\frac{2 l_{2}^{2}(a)\left(1+k^{2}\right)\left(1-k^{2}\right)^{2}-2 z^{2}\left(1+14 k^{2}+k^{4}\right)}{\pi a \rho l_{2}^{3}(a)\left(1-k^{2}\right)^{4}} \mathbf{E}(k), \\
& I(2,0 ; 2)=\frac{2\left\{l_{2}^{2}(a)\left[2 l_{2}^{2}(a)-3 a^{2}\right]\left(1-k^{2}\right)^{2}+z^{2} a^{2}\left(5+3 k^{2}\right)\right\}}{\pi a^{2} l_{2}^{5}(a)\left(1-k^{2}\right)^{3}} \mathbf{F}(k) \\
& +\frac{4\left\{l_{2}^{2}(a)\left[2 a^{2}-\rho^{2}-z^{2}\right]\left(1-k^{2}\right)^{2}-4 a^{2} z^{2}\left(1+k^{2}\right)\right\}}{\pi a^{2} l_{2}^{5}(a)\left(1-k^{2}\right)^{4}} \mathbf{E}(k), \\
& I(0,2 ; 2)=\frac{2\left\{l_{2}^{2}(a)\left[2 l_{2}^{2}(a)-3 \rho^{2}\right]\left(1-k^{2}\right)^{2}+z^{2} \rho^{2}\left(5+3 k^{2}\right)\right\}}{\pi \rho^{2} l_{2}^{5}(a)\left(1-k^{2}\right)^{3}} \mathbf{F}(k) \\
& +\frac{4\left\{l_{2}^{2}(a)\left[2 \rho^{2}-a^{2}-z^{2}\right]\left(1-k^{2}\right)^{2}-4 \rho^{2} z^{2}\left(1+k^{2}\right)\right\}}{\pi \rho^{2} l_{2}^{5}(a)\left(1-k^{2}\right)^{4}} \mathbf{E}(k), \\
& I(1,2 ; 2)=-\frac{2 z\left\{2 l_{2}^{2}(a)\left(1-k^{2}\right)^{2}+7 \rho^{2} k^{2}+\rho^{2}-5 l_{1}^{2}(a)-3 l_{1}^{2}(a) k^{2}\right\}}{\pi a \rho^{2} l_{2}^{3}(a)\left(1-k^{2}\right)^{3}} \mathbf{F}(k) \\
& +\frac{2 z\left\{2\left(1+k^{2}\right)\left[l_{2}^{2}(a)-6 l_{1}^{2}(a)+l_{1}^{2}(a) k^{2}\right]+\rho^{2}\left[1+k^{4}+14 k^{2}\right]\right\}}{\pi a \rho^{2} l_{2}^{3}(a)\left(1-k^{2}\right)^{4}} \mathbf{E}(k),
\end{aligned}
$$




$$
\begin{aligned}
I(2,1 ; 2)= & -\frac{2 z\left\{2 l_{2}^{2}(a)\left(1-k^{2}\right)^{2}+7 a^{2} k^{2}+a^{2}-5 l_{1}^{2}(a)-3 l_{1}^{2}(a) k^{2}\right\}}{\pi a^{2} \rho l_{2}^{3}(a)\left(1-k^{2}\right)^{3}} \mathbf{F}(k) \\
& +\frac{2 z\left\{2\left(1+k^{2}\right)\left[l_{2}^{2}(a)-6 l_{1}^{2}(a)+l_{1}^{2}(a) k^{2}\right]+a^{2}\left[1+k^{4}+14 k^{2}\right]\right\}}{\pi a^{2} \rho l_{2}^{3}(a)\left(1-k^{2}\right)^{4}} \mathbf{E}(k), \\
I(2,2 ; 2)= & \frac{2\left\{\left(1-k^{2}\right)^{2}\left[2 z^{2}+2 l_{2}^{2}(a)-l_{1}^{2}(a)\right]-z^{2} k^{2}\left[5+3 k^{2}\right]\right\}}{\pi a^{2} \rho^{2} l_{2}(a)\left(1-k^{2}\right)^{3}} \mathbf{F}(k) \\
& +\frac{4\left\{4 z^{2} k^{2}\left(1+k^{2}\right)-\left(1-k^{2}\right)^{2}\left[l_{2}^{2}(a)-l_{1}^{2}(a)+k^{2} l_{1}^{2}(a)+z^{2}+z^{2} k^{2}\right]\right\}}{\pi a^{2} \rho^{2} l_{2}(a)\left(1-k^{2}\right)^{4}} \mathbf{E}(k) .
\end{aligned}
$$

12. Evaluations in terms of a Legendre function. Some integrals of the type considered above have also been evaluated in terms of a Legendre function. From Erdelyi (1954) or Byrd and Friedman (1971) one can find

$$
I(\gamma, \gamma ; 0)=\int_{0}^{\infty} J_{\gamma}(a \xi) J_{\gamma}(\rho \xi) e^{-\xi z} d \xi=\frac{1}{\pi(a \rho)^{1 / 2}} Q_{\gamma-1 / 2}(Z)
$$

where $Q_{\gamma-1 / 2}(Z)$ is a Legendre function of the second kind with $Z$ defined as

$$
Z=\frac{a^{2}+\rho^{2}+z^{2}}{2 a \rho}=\frac{l_{1}^{2}(a)+l_{2}^{2}(a)}{2 l_{1}(a) l_{2}(a)}=\frac{1+k^{2}}{2 k}, \quad 1<Z<\infty .
$$

Taking $\gamma=0,1$, and 2 provides the results (Byrd and Friedman, 1971)

$$
\begin{aligned}
& I(0,0 ; 0)=\frac{1}{\pi(a \rho)^{1 / 2}} Q_{-1 / 2}(Z) \\
& I(1,1 ; 0)=\frac{1}{\pi(a \rho)^{1 / 2}} Q_{1 / 2}(Z) \\
& I(2,2 ; 0)=\frac{1}{\pi(a \rho)^{1 / 2}} Q_{3 / 2}(Z) .
\end{aligned}
$$

Comparing the expressions for $I(0,0 ; 0)$ and $I(1,1 ; 0)$ to the results in Sec. 7 provides the relations

$$
\mathbf{F}(k)=\frac{1}{2 \sqrt{k}} Q_{-1 / 2}(Z), \quad \mathbf{E}(k)=\frac{1}{2 \sqrt{k}} Q_{-1 / 2}(Z)-\frac{\sqrt{k}}{2} Q_{1 / 2}(Z)
$$

Using the above equations, all the remaining integral evaluations in Sec. 7 can be 
written in terms of $Q_{-1 / 2}(Z), Q_{1 / 2}(Z)$, and $\Pi(n, k)$. The results are

$$
\begin{aligned}
& I(0,0 ; 1)=\frac{z}{\pi(a \rho)^{1 / 2} l_{2}^{2}(a)\left(1-k^{2}\right)^{2}}\left[\left(1+k^{2}\right) Q_{-1 / 2}(Z)-2 k Q_{1 / 2}(Z)\right], \\
& I(1,0 ; 0)=-\frac{z}{\pi a(a \rho)^{1 / 2}} Q_{-1 / 2}(Z)+\frac{2 z}{\pi a l_{2}(a)} \Pi(n, k), \\
& I(0,1 ; 0)=\frac{1}{\rho}\left[1-\frac{2 z}{\pi l_{2}(a)} \Pi(n, k)\right] \\
& I(0,1 ;-1)=-\frac{z}{\rho}+\frac{1}{\pi(a \rho)^{1 / 2}}\left[\rho Q_{-1 / 2}(Z)-a Q_{1 / 2}(Z)\right]+\frac{2 z^{2}}{\pi \rho l_{2}(a)} \Pi(n, k), \\
& I(1,0 ;-1)=\frac{1}{\pi a(a \rho)^{1 / 2}}\left[\left(a^{2}+z^{2}\right) Q_{-1 / 2}(Z)-a \rho Q_{1 / 2}(Z)\right]-\frac{2 z^{2}}{\pi a l_{2}(a)} \Pi(n, k), \\
& I(1,0 ; 1)=\frac{1}{\pi(a \rho)^{1 / 2} l_{2}^{4}(a)\left(1-k^{2}\right)^{2}}\left[a\left(a^{2}+z^{2}-\rho^{2}\right) Q_{-1 / 2}(Z)\right. \\
& I(0,1 ; 1)=\frac{1}{\pi(a \rho)^{1 / 2} l_{2}^{4}(a)\left(1-k^{2}\right)^{2}}\left[\rho\left(\rho^{2}+z^{2}-a^{2}\right) Q_{-1 / 2}(Z)\right. \\
& I(1,1 ; 1)\left.=\frac{z}{\left.\pi(a \rho)^{3 / 2}\left(1-k^{2}\right) Q_{1 / 2}(Z)\right],}\left[2 k^{2}+z^{2}-\rho^{2}\right) Q_{1 / 2}(Z)\right], \\
& I(1,1 ;-1)=\frac{a}{2 \rho}-\frac{z\left(a^{2}-\rho^{2}\right)}{2 \pi a(a \rho)^{1 / 2}}\left[\rho Q_{-1 / 2}(Z)+a Q_{1 / 2}(Z)\right]-\frac{z}{\pi a \rho l_{2}(a)} \Pi(n, k), \\
&\left.I\left(1+k^{2}\right) Q_{1 / 2}(Z)\right] .
\end{aligned}
$$

It was noted in the introduction that Hasegawa et al. (1992a,b) evaluated the integrals considered presently in terms of a Legendre function. However, his choice of the parameters in the complete elliptic integral of the third kind was consistent with that used by Eason et al. (1955). The integral evaluations given by Hasegawa et al. (1992b) were shown to agree with the above equations using the transformation formula in Eq. (24).

13. Evaluations on the axis $\rho=0$ and at the surface $z=0$. In physical problems where these integrals may appear as the solution for some field quantity, $\rho$ would usually represent the cylindrical coordinate radius measured from the $z$-axis where $z \geq 0$ for convergence. It might therefore be required to evaluate the above integral evaluations at $\rho=0$ or $z=0$.

Since $\rho$ appears in the denominator of many of the above expressions, the individual terms in a particular integral evaluation become infinite. However, these infinities cancel when all terms in an expression are considered. To obtain the analytical expression for these integrals when $\rho=0$, one could use the power series expansions for the elliptic integrals given in Sec. 8 of Hanson and Puja (1996). An easier approach is available however. To this end we return to the definition for $I(\mu, \nu ; \lambda)$ given in Eq. (1). The function $J_{\nu}(\rho \xi)$ can be expanded in a power series in $\rho$ and the resulting integrals evaluated very 
easily. This procedure leads to the $\rho \rightarrow 0$ expansions given in Appendix A for the various integers $\mu, \nu$, and $\lambda$.

To evaluate these integrals when $z \rightarrow 0$, the following limits can be used:

$$
\operatorname{Lim}_{z \rightarrow 0} l_{1}(a)=\min (a, \rho), \quad \operatorname{Lim}_{z \rightarrow 0} l_{2}(a)=\max (a, \rho)
$$

where min is the minimum of the two values and max is the maximum. Thus $k$ and $n$ become

$$
\begin{array}{ll}
k=\frac{\rho}{a}, \quad \rho<a ; & k=\frac{a}{\rho}, \quad \rho>a, \\
n=1, \quad \rho<a ; & n=\frac{a^{2}}{\rho^{2}}, \quad \rho>a .
\end{array}
$$

The only difficulty in evaluating the expressions at the surface results from the complete elliptic integral of the third kind. From Eq. (12), it is seen that when $z \rightarrow 0$ with $\rho<a(n \rightarrow 1)$, this elliptic integral tends to infinity. However, in the integral evaluations above, this elliptic integral is multiplied by $z$ which tends to zero. To evaluate this limit, Eq. (24) can be used providing the result

$$
\operatorname{Lim}_{z \rightarrow 0} z \Pi(n, k)=\frac{\pi a}{2}, \quad \rho<a ; \quad \operatorname{Lim}_{z \rightarrow 0} z \Pi(n, k)=0, \quad \rho>a .
$$

From the above three equations, the values of any of the integrals can be found for $z \rightarrow 0$.

14. Discussion and conclusions. In this paper certain infinite integrals involving products of Bessel functions of integer order have been evaluated. These integrals have been investigated and evaluated previously by Eason et al. (1955). They used an integral representation for $I(\mu, \nu ; \lambda)$ in terms of a hypergeometric function which allowed the evaluation in terms of elliptic integrals for various integer values of the parameters. This evaluation procedure led naturally to a certain definition of the modulus $k_{s}$ and parameter $p$ in their elliptic integrals. This choice of parameters and the use of Heuman's Lambda function led to evaluations for some of the integrals that had different forms depending on the relative magnitudes of $\rho$ and $a$. Since these integral evaluations are generally some physical quantity such as displacement or stress fields in linear elasticity, the requirement of needing different expressions in different regions to represent continuously differentiable functions is a bit disconcerting.

In a recent paper Hanson and Puja (1996) evaluated the elastic field for a uniform normal or shear loading on a transversely isotropic half space. Following Love's (1929) method of solution, they determined the elastic fields by directly evaluating the double integral of the potential function. Since this problem could also be formulated using Hankel transforms, the integral evaluations in their paper could be used to directly evaluate some of the integrals considered here (as done in Sec. 7). However, their alternative method of evaluation led to new forms for the elliptic modulus $k$ and parameter $n$. This new form has allowed $I(\mu, \nu ; \lambda)$ to be evaluated in terms of a single expression, continuous and differentiable everywhere, for all values of the parameters $\rho, z$, and $a$. In some instances this new form has also allowed these integral evaluations to be put in a simpler form. 
As a final point, we note that the present work has correlated the present integral evaluations with those existing in the previous literature. The required transformation formulas were presented in Sec. 5. It was found that all previously evaluated integrals were consistent with the present results.

Acknowledgment. It is gratefully acknowledged that support during the course of this research was received from the National Science Foundation under grant No. MSS9210531.

Appendix A. Many of the integral evaluations derived in this paper contain the polar radius $\rho$ in the denominator. This may lead to an indeterminacy in trying to evaluate some of the expressions at the origin $\rho=0$. Below the asymptotic expressions are given for each integral as $\rho$ tends to zero. These limits can be obtained from the results in Hanson and Puja (1996). However, they are presently obtained by expanding the Bessel function $J_{\nu}(\rho \xi)$ for $\rho \rightarrow 0$ and evaluating the resulting integral. The results are

$$
\begin{aligned}
& I(0,1 ;-1)=\frac{1}{2\left(a^{2}+z^{2}\right)^{1 / 2}} \rho+O\left(\rho^{3}\right), \\
& I(1,0 ;-1)=\frac{\left(a^{2}+z^{2}\right)^{1 / 2}-z}{a}+O\left(\rho^{2}\right), \\
& I(1,1 ;-1)=\frac{\left[\left(a^{2}+z^{2}\right)^{1 / 2}-z\right]}{2 a\left(a^{2}+z^{2}\right)^{1 / 2}} \rho+O\left(\rho^{3}\right), \\
& I(0,0 ; 0)=\frac{1}{\left(a^{2}+z^{2}\right)^{1 / 2}}+O\left(\rho^{2}\right), \\
& I(0,1 ; 0)=\frac{z}{2\left(a^{2}+z^{2}\right)^{3 / 2}} \rho+O\left(\rho^{3}\right), \\
& I(1,0 ; 0)=\frac{\left(a^{2}+z^{2}\right)^{1 / 2}-z}{a\left(a^{2}+z^{2}\right)^{1 / 2}}+O\left(\rho^{2}\right), \\
& I(1,1 ; 0)=\frac{a}{2\left(a^{2}+z^{2}\right)^{3 / 2}} \rho+O\left(\rho^{3}\right), \\
& I(0,0 ; 1)=\frac{z}{\left(a^{2}+z^{2}\right)^{3 / 2}}+O\left(\rho^{2}\right), \\
& I(0,1 ; 1)=\frac{\left[2 z^{2}-a^{2}\right]}{2\left(a^{2}+z^{2}\right)^{5 / 2}} \rho+O\left(\rho^{3}\right), \\
& I(1,0 ; 1)=\frac{a}{\left(a^{2}+z^{2}\right)^{3 / 2}}+O\left(\rho^{2}\right), \\
& I(1,1 ; 1)=\frac{3 z a}{2\left(a^{2}+z^{2}\right)^{5 / 2}} \rho+O\left(\rho^{3}\right), \\
& I(0,2 ;-1)=\frac{z}{8\left(a^{2}+z^{2}\right)^{3 / 2}} \rho^{2}+O\left(\rho^{4}\right), \\
& I(2,0 ;-1)=\frac{a^{2}+2 z^{2}-2 z\left(a^{2}+z^{2}\right)^{1 / 2}}{2 a^{2}}+O\left(\rho^{2}\right), \\
& I(1,2 ;-1)=\frac{a}{8\left(a^{2}+z^{2}\right)^{3 / 2}} \rho^{2}+O\left(\rho^{4}\right),
\end{aligned}
$$




$$
\begin{aligned}
& I(2,1 ;-1)=\frac{a^{2}+2 z^{2}-2 z\left(a^{2}+z^{2}\right)^{1 / 2}}{2 a^{2}\left(a^{2}+z^{2}\right)^{1 / 2}} \rho+O\left(\rho^{3}\right), \\
& I(2,2 ;-1)=\frac{2\left(a^{2}+z^{2}\right)^{3 / 2}-3 z a^{2}-2 z^{3}}{8 a^{2}\left(a^{2}+z^{2}\right)^{3 / 2}} \rho^{2}+O\left(\rho^{4}\right), \\
& I(0,2 ; 0)=\frac{\left[2 z^{2}-a^{2}\right]}{8\left(a^{2}+z^{2}\right)^{5 / 2}} \rho^{2}+O\left(\rho^{4}\right), \\
& I(2,0 ; 0)=\frac{a^{2}+2 z^{2}-2 z\left(a^{2}+z^{2}\right)^{1 / 2}}{a^{2}\left(a^{2}+z^{2}\right)^{1 / 2}}+O\left(\rho^{2}\right), \\
& I(1,2 ; 0)=\frac{3 z a}{8\left(a^{2}+z^{2}\right)^{5 / 2}} \rho^{2}+O\left(\rho^{4}\right), \\
& I(2,1 ; 0)=\frac{2\left(a^{2}+z^{2}\right)^{3 / 2}-3 z a^{2}-2 z^{3}}{2 a^{2}\left(a^{2}+z^{2}\right)^{3 / 2}} \rho+O\left(\rho^{3}\right), \\
& I(2,2 ; 0)=\frac{3 a^{2}}{8\left(a^{2}+z^{2}\right)^{5 / 2}} \rho^{2}+O\left(\rho^{4}\right), \\
& I(0,2 ; 1)=\frac{z\left[6 z^{2}-9 a^{2}\right]}{8\left(a^{2}+z^{2}\right)^{7 / 2}} \rho^{2}+O\left(\rho^{4}\right), \\
& I(2,0 ; 1)=\frac{2\left(a^{2}+z^{2}\right)^{3 / 2}-3 z a^{2}-2 z^{3}}{a^{2}\left(a^{2}+z^{2}\right)^{3 / 2}}+O\left(\rho^{2}\right), \\
& I(1,2 ; 1)=\frac{3 a\left[4 z^{2}-a^{2}\right]}{8\left(a^{2}+z^{2}\right)^{7 / 2}} \rho^{2}+O\left(\rho^{4}\right), \\
& I(2,1 ; 1)=\frac{3 a^{2}}{2\left(a^{2}+z^{2}\right)^{5 / 2}} \rho+O\left(\rho^{3}\right), \\
& I(2,2 ; 1)=\frac{15 z a^{2}}{8\left(a^{2}+z^{2}\right)^{7 / 2}} \rho^{2}+O\left(\rho^{4}\right), \\
& I(1,1 ;-2)=\frac{\left(a^{2}+z^{2}\right)^{1 / 2}-z}{2 a} \rho+O\left(\rho^{3}\right), \\
& I(2,0 ;-2)=\frac{2\left(a^{2}+z^{2}\right)^{3 / 2}-3 z a^{2}-2 z^{3}}{6 a^{2}}+O\left(\rho^{2}\right), \\
& I(0,2 ;-2)=\frac{1}{8\left(a^{2}+z^{2}\right)^{1 / 2}} \rho^{2}+O\left(\rho^{4}\right), \\
& I(1,2 ;-2)=\frac{\left(a^{2}+z^{2}\right)^{1 / 2}-z}{8 a\left(a^{2}+z^{2}\right)^{1 / 2}} \rho^{2}+O\left(\rho^{4}\right), \\
& I(2,1 ;-2)=\frac{a^{2}+2 z^{2}-2 z\left(a^{2}+z^{2}\right)^{1 / 2}}{4 a^{2}} \rho+O\left(\rho^{3}\right), \\
& I(2,2 ;-2)=\frac{a^{2}+2 z^{2}-2 z\left(a^{2}+z^{2}\right)^{1 / 2}}{8 a^{2}\left(a^{2}+z^{2}\right)^{1 / 2}} \rho^{2}+O\left(\rho^{4}\right), \\
& I(0,0 ; 2)=\frac{2 z^{2}-a^{2}}{\left(a^{2}+z^{2}\right)^{5 / 2}}+O\left(\rho^{2}\right), \\
& I(1,0 ; 2)=\frac{3 a z}{\left(a^{2}+z^{2}\right)^{5 / 2}}+O\left(\rho^{2}\right),
\end{aligned}
$$




$$
\begin{aligned}
& I(0,1 ; 2)=\frac{z\left[6 z^{2}-9 a^{2}\right]}{2\left(a^{2}+z^{2}\right)^{7 / 2}} \rho+O\left(\rho^{3}\right), \\
& I(1,1 ; 2)=\frac{3 a\left[4 z^{2}-a^{2}\right]}{2\left(a^{2}+z^{2}\right)^{7 / 2}} \rho+O\left(\rho^{3}\right), \\
& I(2,0 ; 2)=\frac{3 a^{2}}{\left(a^{2}+z^{2}\right)^{5 / 2}}+O\left(\rho^{2}\right), \\
& I(0,2 ; 2)=\frac{24 z^{4}+9 a^{4}-72 a^{2} z^{2}}{8\left(a^{2}+z^{2}\right)^{9 / 2}} \rho^{2}+O\left(\rho^{4}\right), \\
& I(1,2 ; 2)=\frac{15 a z\left[4 z^{2}-3 a^{2}\right]}{8\left(a^{2}+z^{2}\right)^{9 / 2}} \rho^{2}+O\left(\rho^{4}\right), \\
& I(2,1 ; 2)=\frac{15 z a^{2}}{2\left(a^{2}+z^{2}\right)^{7 / 2}} \rho+O\left(\rho^{3}\right), \\
& I(2,2 ; 2)=\frac{15 a^{2}\left[6 z^{2}-a^{2}\right]}{8\left(a^{2}+z^{2}\right)^{9 / 2}} \rho^{2}+O\left(\rho^{4}\right) .
\end{aligned}
$$

\section{REFERENCES}

[1] P. F. Byrd and M. D. Friedman, Handbook of Elliptic Integrals for Engineers and Scientists, Springer-Verlag, 1971, pp. 13-14, 35-41, 249-250

[2] G. Eason, B. Noble, and I. N. Sneddon, On certain integrals of Lipschitz-Hankel type involving products of Bessel functions, Philos. Trans. Roy. Soc. London A247, 529-551 (1955)

[3] A. Erdelyi, Tables of Integral Transforms, Vol. 2, McGraw-Hill, 1954, p. 50

[4] V. I. Fabrikant, Applications of Potential Theory in Mechanics: a selection of new results, Kluwer Academic Publishers, The Netherlands, 1989, pp. 9-17

[5] I. S. Gradshteyn and I. M. Ryzhik, Table of Integrals, Series, and Products, Academic Press, 1980, pp. $707,904-909$

[6] M. T. Hanson and I. W. Puja, Love's circular patch problem revisited: Closed form solution for transverse isotropy and shear loading, Quart. Appl. Math. 54, 359-384 (1996)

[7] H. Hasegawa, V. Lee, and T. Mura, Green's functions for axisymmetric problems of dissimilar elastic solids, ASME J. Appl. Mech. 59, S107-S114 (1992)

[8] H. Hasegawa, V. Lee, and T. Mura, The stress fields caused by a circular cylindrical inclusion, ASME J. Appl. Mech. 59, 312-320 (1992)

[9] C. Heuman, Tables of complete elliptic integrals, Journal of Math. Phys., Vol. 20, 1941, pp. 127-206

[10] A. E. H. Love, The stress produced in a semi-infinite solid by pressure on part of the boundary, Philos. Trans. Roy. Soc. London A228, 377-420 (1929)

[11] R. Muki, Asymmetric problems of the theory of elasticity for a semi-infinite solid and a thick plate, Progress in Solid Mechanics (I. N. Sneddon and R. Hill, eds.), North Holland, Amsterdam, 1960, pp. 399-439

[12] I. N. Sneddon, Fourier Transforms, McGraw-Hill, 1951, pp. 450-473

[13] I. N. Sneddon, The Use of Integral Transforms, McGraw-Hill, 1972, pp. 325-352

[14] M. R. Sura-Bura, Doklady Akad. Nauk S.S.S.R. (n.s.) 73, 901 (1950)

[15] K. Terazawa, On the elastic equilibrium of a semi-infinite solid, J. College Sci., University of Tokyo, Vol. 37, article 7, 1916

[16] G. N. Watson, A Treatise on the Theory of Bessel Functions, Cambridge University Press, 1980, p. 358 